1 Universidade Federal do Sul da Bahia (UFSB) Itabuna (BA), Brasil. Orcid: https://orcid org/0000-0002-95382675

janemg@ufsb.edu.br

2 Universidade Federal da Bahia (UFBA), Instituto de Saúde Coletiva (ISC) Salvador (BA), Brasil. Orcid:https://orcid. org/0000-0002-49030556

erikapecs@gmail.com

${ }^{3}$ Centro Universitário Estácio da Bahia - Salvador (BA), Brasil.

Orcid: https://orcid. org/0000-0003-35206757

fabol78@gmail.com

4 Fundação Oswaldo Cruz (Fiocruz) - Salvador (BA) Brasil.

Orcid: https://orcid. org/0000-0001-8918-

2661

bethania.almeida@fiocruz.br

5 Universidade Federal da Bahia (UFBA), Instituto de Saúde Coletiva (ISC) Salvador (BA), Brasil. Orcid: https://orcid. org/0000-0002-45404944

loureiro9301@gmail.com

\section{Paradigmas e trajetórias tecnológicas em saúde: desafios da inovação no cuidado da diabetes}

\author{
Technological paradigms and trajectories in health: challenges of \\ innovation in the care of diabetes

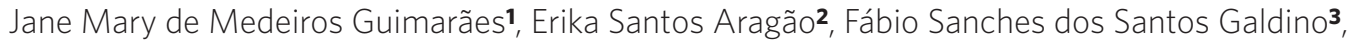 \\ Bethania de Araújo Almeida ${ }^{4}$, Sebastião Antonio Loureiro de Souza Silva ${ }^{5}$
}

DOI: 10.1590/0103-11042018S215

RESUMO No setor da saúde, a incorporação de inovações tem contribuído para um aumento da demanda por serviços públicos, bem como por tecnologias e materiais utilizados na prestação de serviços de saúde. Um dos segmentos que mais pressionam os serviços em termos da incorporação de seus produtos é a indústria farmacêutica. Este estudo tem como objetivo descrever as trajetórias tecnológicas no segmento de medicamentos para a diabetes lançados no Brasil entre 1998 e 2012. Essas drogas representam uma importante proporção de gastos com medicamentos no País. Ao empregar uma metodologia qualitativa, descritiva e exploratória, este estudo buscou responder às seguintes perguntas: houve mudanças nas trajetórias tecnológicas aqui consideradas ao longo do referido período de tempo? As inovações incorporadas no País têm sido de natureza incremental ou radical? Quais são as evidências que apoiam as mudanças de direção? À luz dos dados coletados a partir de uma variedade de bases de dados, foi possível verificar que os medicamentos contra a diabetes introduzidos durante esse período representaram inovações incrementais em relação às trajetórias tecnológicas aqui consideradas.

PALAVRAS-CHAVE Inovação. Difusão de inovações. Diabetes Mellitus. Hipoglicemiantes.

ABSTRACT In the health sector, the incorporation of innovations has contributed to an increased demand for public services, as well as technologies and the supplies used in the provision of health services. One of the health sectors which causes most pressures on services in terms of the incorporation of its products is the pharmaceutical industry. This study aims to describe the technological trajectories in the segment of anti-diabetic medication launched in Brazil between 1998 and 2012. These drugs represent a significant proportion of spending on medicine in the Country. By using a qualitative, descriptive and exploratory methodology, the present study endeavored to answer the following questions: have changes taken place in the technological trajectories over the time period considered herein? Have the innovations incorporated in the Country been of an incremental or radical nature? What are the evidences that support these changes in direction? In light of the data collected from a variety of databases, it was possible to verify that the anti-diabetic medications introduced during this period represented incremental innovations with respect to the technological trajectories considered herein.

KEYWORDS Innovation. Diffusion of innovation, Diabetes Mellitus. Hypoglycemic Agents 


\section{Introdução}

Em saúde, as inovações representam a aplicação de novos conhecimentos, que podem aparecer de forma concretamente incorporada num artefato físico; ou seja, num equipamento, dispositivo ou medicamento, e também podem representar 'ideias', na forma de novos procedimentos ou de reorganização dos serviços ${ }^{1}$.

Para Dosi², a inovação tecnológica é um instrumento de concorrência que gera uma vantagem competitiva de custos ou qualidade, podendo ser real ou simbólica para o inovador. Portanto, é um processo heterogêneo e complexo, e sua dinâmica difere entre países, setores e empresas, o que torna a inovação uma variável fundamental para o desenvolvimento econômico e social.

Na saúde, a incorporação dessas inovações tem contribuído para o aumento das demandas por serviços públicos, tecnologias e insumos no sistema de saúde. Essas fazem crescer o orçamento nacional destinado ao setor. Em países como o Brasil, com problemas macroeconômicos e estruturais, é constante a preocupação com a otimização dos recursos públicos, que são escassos, particularmente, no contexto de um sistema universal de saúde, no qual toda população tem direito a ações e serviços de saúde. Logo, o atendimento das necessidades de saúde da população exige do Estado mecanismos de regulação complexos e muitas vezes sofisticados para o cuidado e a promoção da saúde e para a redução de riscos e agravos.

Numa estrutura de mercado oligopolista do setor, em que a inovação é o principal elemento de diferenciação, a indústria farmacêutica possui elevadas barreiras em função da proteção patentária, que garante o monopólio temporário ao seu detentor. Isso pressupõe elevados investimentos em Pesquisa e Desenvolvimento (P\&D), controle do fornecimento de princípios ativos e força mercadológica, através de pesados investimentos em marketing. Esse setor apresenta quatro estágios tecnológicos que definem o grau de domínio do processo produtivo, sendo: a) pesquisa e desenvolvimento de novos fármacos; b) produção de novos fármacos; c) pesquisa clínica e produção de medicamentos; e d) marketing e comercialização.

A Diabetes Mellitus (DM) foi escolhida em decorrência de sua prevalência estar aumentando no Brasil e por se configurar como uma epidemia mundial, traduzindo-se em grande desafio para os sistemas de saúde de todo o mundo. Registram-se quatro milhões de mortes por ano relativas à diabetes e às suas complicações, o que representa $9 \%$ da mortalidade mundial total. $\mathrm{O}$ grande impacto econômico ocorre notadamente nos serviços de saúde, como consequência dos crescentes custos do tratamento da doença ${ }^{3}$.

Desde que foi descoberta, a diabetes passou a ser objeto de estudos científicos e de interesse das firmas na produção de medicamentos. Os avanços no conhecimento, na terapia e na tecnologia tornaram mais eficiente o cuidado dos portadores de DM. Medicamentos, insumos e procedimentos fazem parte do processo inovador, no âmbito da saúde, tendo a insulina sido a grande descoberta, em 1922. Desde então, uma série de esforços tem sido empreendida para diagnóstico, controle e tratamento da doença, ou das 'doenças'.

A partir das propostas de Marx sobre o papel da tecnologia no funcionamento da economia capitalista, Schumpeter ${ }^{4}$ produziu sua Teoria do Desenvolvimento com base no conceito de monopólio temporário do inovador. A inovação passa a ser considerada imprescindível na promoção da competitividade e do progresso social. Portanto, a mudança tecnológica é compreendida por Schumpeter ${ }^{4}$ como o motor do desenvolvimento capitalista; a firma, o locus de atuação do empresário inovador e de desenvolvimento de inovações.

A pressão pela incorporação das tecnologias geradas na indústria farmacêutica, particularmente, tem se traduzido em gastos crescentes dos sistemas de saúde, sobretudo 
com o advento da biotecnologia. Ao aplicar conhecimentos da biologia molecular, biologia celular, bioquímica, fisiologia, farmacologia e de outras disciplinas afins, a indústria farmacêutica passou a conviver com duas trajetórias.

A primeira trajetória, com origem no final do século XIX, é baseada na química fina, que possibilitou a criação dos fármacos tradicionais; a segunda emergiu nos anos 1970, a partir da descoberta da estrutura do Ácido Desoxirribonucleico (DNA) (nos anos 1950) e, posteriormente, das técnicas de modificação dessa estrutura, fortemente baseadas na genômica, proteômica e DNA recombinante ${ }^{5-7}$. As técnicas genômicas estudam o conjunto dos genes e as técnicas proteômica buscam compreender as redes funcionais que são estabelecidas entre as proteínas codificadas pelos genes.

Optou-se por acompanhar as trajetórias tecnológicas, ou seja, a direção tomada pelo desenvolvimento tecnológico dos medicamentos utilizados no controle da DM. De acordo com o Ministério da Saúde ${ }^{3(16)}$,

a diabetes é um grupo de doenças metabólicas caracterizadas por hiperglicemia e associadas a complicações, disfunções e insuficiência de vários órgãos, especialmente olhos, rins, nervos, cérebro, coração e vasos sanguíneos. Pode resultar de defeitos de secreção e/ou ação da insulina, envolvendo processos patogênicos específicos, por exemplo, destruição das células beta do pâncreas (produtoras de insulina), resistência à ação da insulina, distúrbios da secreção da insulina, entre outros.

Por ser uma doença crônica, que evolui com o decorrer dos anos. Quase todos os pacientes fazem tratamento farmacológico; muitos deles, com o uso de insulina.

Esse corte se justifica pelos avanços da regulação no setor farmacêutico no Brasil e pela criação da Agência Nacional de Vigilância Sanitária (Anvisa), em 1999, que marca o início da regulação em saúde, sob a forma de agências que atuam em todos os setores que estão relacionados a produtos e a serviços que possam afetar a saúde da população brasileira.

$\mathrm{Na}$ abordagem neo-schumpteriana (evolucionária), o progresso técnico é um elemento que afeta o processo de crescimento econômico ao introduzir transformações nos sistemas socioeconômicos, que alteram as estratégias produtivas das empresas. Essas transformações são condicionadas por aspectos internos e aspectos externos.

Na busca da prevenção e do cuidado, novas tecnologias são disponibilizadas nos serviços como opções terapêuticas, que visam a prevenir ou retardar as complicações agudas e crônicas que podem se tornar um sério problema de saúde pública, pois pioram a qualidade de vida dos portadores de diabetes.

O objetivo deste estudo é o de analisar as trajetórias tecnológicas dos medicamentos para o controle da diabetes lançados no Brasil, no período entre 1998 e 2012.

\section{Material e métodos}

\section{Marco Teórico}

Paradigmas são definidos por Thomas $\mathrm{Kuhn}^{\mathbf{8 ( 1 6 )}}$ como

realizações científicas universalmente reconhecidas que, durante algum tempo, fornecem problemas e soluções modelares para uma comunidade de praticantes de uma ciência.

No âmbito econômico, Dosi² relaciona o conceito de paradigma tecnológico, em ampla analogia, com o conceito de paradigma científico de Kuhn. Para ele, o paradigma tecnológico é caracterizado pelo conjunto de métodos e regras que conduzem da descoberta à resolução dos problemas. Trata-se de um padrão normal de atividades, circunscrito aos limites do paradigma. A mudança de um paradigma geralmente implica a mudança da trajetória tecnológica ${ }^{2}$. 
A trajetória tecnológica estabelece um agrupamento de possíveis direções a serem seguidas. Pode haver trajetórias mais poderosas, a depender do conjunto de tecnologias excluído por essa trajetória. Existe, ainda, a possibilidade do progresso numa trajetória, como a de conservar aspectos cumulativos. Portanto, futuros avanços dependem das tendências tecnológicas desenvolvidas pelas empresas ou pelo País, e que já ocupam a fronteira tecnológica.

Nessa perspectiva, as inovações radicais, ou seja, as inovações que constituem a entrada de algo realmente novo no mercado, como uma nova molécula ou um novo modelo de produção (biotecnologia moderna), contribuem para a construção de trajetórias, podendo, inclusive, como no segundo caso, constituir um paradigma tecnológico. As inovações incrementais, por seu turno, são muito importantes, tanto do ponto de vista econômico quanto social, mas constituem apenas melhorias ou modificações de bens ou processos existentes.

\section{Estratégia empírica}

Para acompanhar as trajetórias tecnológicas adotadas no desenvolvimento dos medicamentos utilizados para o controle da diabetes, foi aplicada uma metodologia de natureza qualitativa, descritiva, exploratória. Para compreender melhor a inserção desse conceito no setor saúde, optou-se por acompanhar os antidiabéticos lançados no Brasil, no período de 1998 a 2012.

O recorte temporal definido decorre do fato de que, em 1998, foi publicada a Política Nacional de Medicamentos (PNM), que definiu diretrizes para garantir a segurança, a eficácia e a qualidade dos medicamentos, o seu uso racional e $o$ acesso da população aos medicamentos considerados essenciais. Além disso, em 1999, foi criada a Lei no 9.787/1999 (Lei dos Genéricos), que reduziu o preço médio dos medicamentos nos mercados.

Inicialmente, foi realizado um levantamento bibliográfico na base de dados da
Biblioteca Virtual em Saúde (BVS). Utilizouse, na busca, o descritor Diabetes Mellitus; ano de publicação, entre 1998 e 2012; restrita a humanos; e texto completo disponível. Foram analisados 108 artigos acerca das tecnologias envolvidas no controle da diabetes. Posteriormente, foi realizada uma nova busca na BVS e na Scientific Electronic Library Online (SciELO), a partir dos descritores: insulina, hipoglicemiantes, medicamentos. Do total de 24 artigos, oito foram selecionados, os que se referiam ao tratamento medicamentoso e à sua evolução para o cuidado da diabetes. Na base de dados do Pubmed, foram utilizados os descritores: antidiabetic drugs; insulin therapy; diabetes mellitus; diabetes type 2. A escolha dos artigos seguiu os mesmos critérios.

As informações sobre os fornecedores de antidiabéticos foram levantadas no sistema de avaliação de fármacos lançados (genéricos) na base de dados Newport, da Thomson Reuters ${ }^{\circledR}$. Esta foi consolidada como base de dados para instituições interessadas no mercado de genéricos e similares, medicamentos de venda livre e fabricantes de insumos ativos, para auxiliarem na identificação e na avaliação de produtos em fase de comercialização e oportunidades de licenciamento.

Trata-se de uma base de dados paga e de acesso restrito, onde a busca foi realizada através do Target Products Search, a partir dos seguintes descritores: país onde os produtos foram lançados (Launched Products) - no caso deste estudo, o Brasil; o período de lançamento (Pack Launch Date), de 01/01/1998 até $31 / 12 / 2012$; e a categoria terapêutica escolhida foi EphMRA (A10 - Drugs Used In Diabetes), medicamentos antidiabéticos.

A partir desses critérios de busca, foram extraídos do banco de dados 42 medicamentos lançados no mercado e que possuíam seu princípio ativo relacionado ao tratamento da diabetes tipo 1 e 2 como antidiabéticos. Desse total, sete medicamentos referiam-se ao tratamento de comorbidades, e por isso foram excluídos da análise. 
Os 35 medicamentos, antidiabéticos considerados válidos, foram classificados por grupos farmacológicos e terapêuticos com relação à sua afinidade farmacodinâmica, ou seja, pela ação dos efeitos fisiológicos dos fármacos nos organismos e seus mecanismos de ação.

Para identificar a existência ou não de inovação, os medicamentos foram analisados individualmente a partir do seu princípio ativo. Após essa análise, foi feito um comparativo das inovações ocorridas, por grupo farmacológico. Para isso, foram determinadas as possíveis alterações no princípio ativo, a cada lançamento, para se identificar o tipo de inovação disponibilizada no mercado brasileiro. A despeito de, a partir de 2012, terem sido lançados no mercado novos medicamentos para diabetes, o acesso à base de dados utilizada para levantamento dos fornecedores e tecnologias não estava mais disponível devido ao encerramento da licença. Tal fato se constitui uma limitação do estudo.

\section{Resultados}

Os dados foram produzidos a partir dos resultados apurados na base de dados da Newport ${ }^{\circledR}$. A análise possibilitou verificar os tipos de inovações ocorridas no período analisado, bem como, através da bibliografia disponível, responder sobre a mudança ou não de paradigma para o cuidado da diabetes.

\section{Dinâmica do mercado de antidiabéticos}

Os medicamentos antidiabéticos, lançados no Brasil, foram classificados em oito grupos farmacológicos, conforme gráfico 1, abaixo.

Gráfico 1. Medicamentos por grupos farmacológicos e lançamentos no Brasil

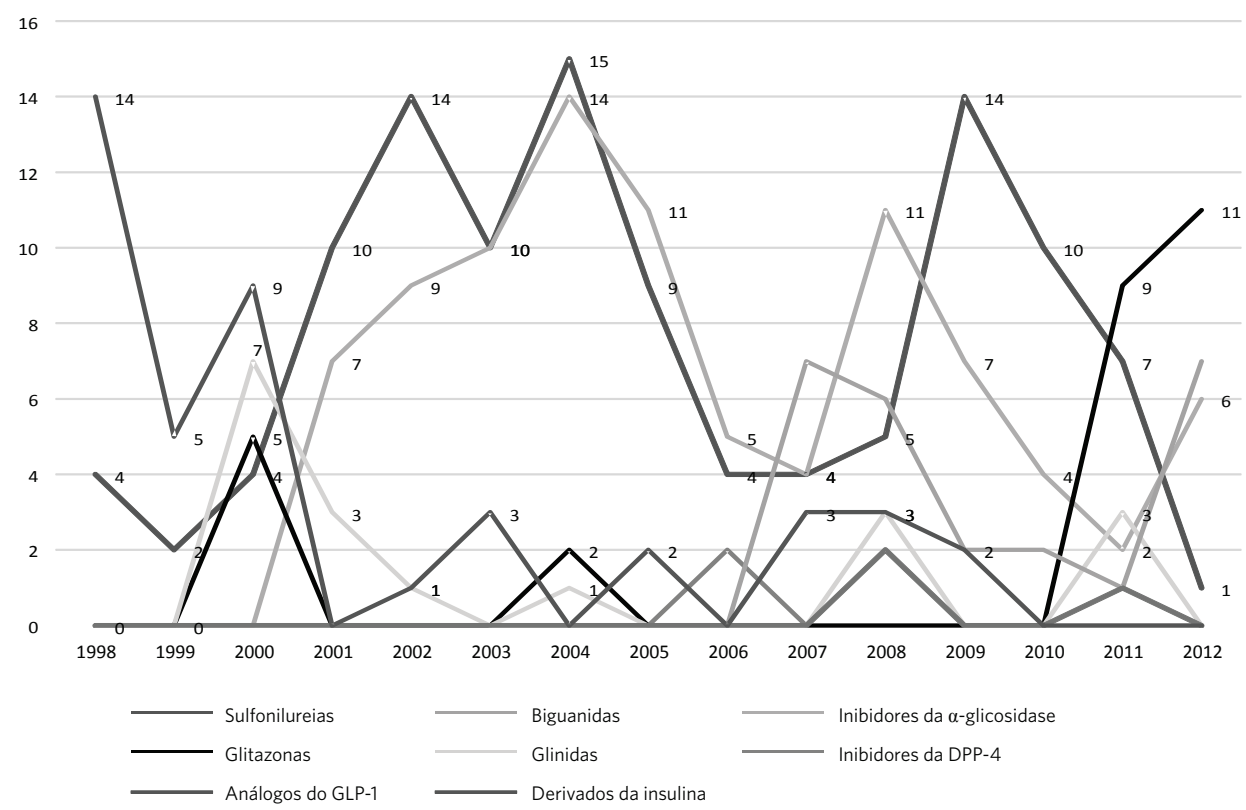

Fonte: Guimarães 9 .

No quadro 1, a seguir, encontram-se relacionados os efeitos terapêuticos dos medicamentos antidiabéticos, por grupo farmacológico. 
Quadro 1. Efeitos terapêuticos dos medicamentos antidiabéticos, por grupo farmacológico

\begin{tabular}{|c|c|}
\hline Grupos farmacológicos & Efeito terapêutico \\
\hline Sulfonilureia & $\begin{array}{l}\text { Ser um secretagogo beta-pancreático de ação lenta que aumenta a secreção de insulina. } \\
\text { Trata-se de um hipoglicemiante, e fazem parte deste grupo os medicamentos: chlorpro- } \\
\text { pamide, gliclazide, glimepiride, glipizide, e glyburide. }\end{array}$ \\
\hline Biguanida & $\begin{array}{l}\text { Diminui a produção hepática de glicose e aumenta a sensibilidade à insulina no músculo } \\
\text { esquelético, no tecido adiposo e, especialmente, no fígado, reduzindo a gliconeogênese } \\
\text { hepática e aumentando a captação periférica de glicose. }\end{array}$ \\
\hline Inibidores da $\alpha$-glicosidase & Retardam a absorção intestinal de carboidratos. Faz parte deste grupo a acarbose. \\
\hline Glitazonas & $\begin{array}{l}\text { Aumenta a sensibilidade à insulina em músculo, adipócito e hepatócito. Os medicamen- } \\
\text { tos que fazem parte deste grupo são: pioglitazone hydrochloride e rosiglitazone maleate. }\end{array}$ \\
\hline Glinidas & $\begin{array}{l}\text { Trata-se de um secretagogo beta-pancreáticos de ação rápida. Estão neste grupo: nate- } \\
\text { glinide e repaglinide. }\end{array}$ \\
\hline $\begin{array}{l}\text { Inibidores da DPP-4 ou } \\
\text { gliptinas }\end{array}$ & $\begin{array}{l}\text { Prolongam a ação dos hormônios insulina e glucagon, inibindo a enzima DPP-4, que } \\
\text { desativa as incretinas. A linagliptin, saxagliptin, hydrochloride monohydrate, sitagliptin } \\
\text { phosphate monohydrate, vildagliptin e sitagliptin phosphate fazem parte deste grupo. }\end{array}$ \\
\hline Análogos do GLP-1 & $\begin{array}{l}\text { Mimetizam a ação dos hormônios GLP-1 secretados pela porção final do intestino del- } \\
\text { gado, promovendo a saciedade e a secreção de insulina. Os medicamentos que fazem } \\
\text { parte deste grupo são: exenatide e liraglutide. }\end{array}$ \\
\hline Derivados da insulina & $\begin{array}{l}\text { Tecnologia aplicada na produção de insulina artificial. Aumenta seu perfil absortivo e metabó- } \\
\text { lico. Trata-se de um grupo com grande número de medicamentos, sendo eles: insulin aspart, } \\
\text { insulin detemir, insulin glargine, insulin glulisine recombinant, insulin human recombinant, } \\
\text { insulin isophane recombinant human, insulin lispro recombinant. }\end{array}$ \\
\hline
\end{tabular}

Fonte: Guimarães 9

O gráfico 2 apresenta a associação entre farmacológicos. diferentes medicamentos, por grupos

Gráfico 2. Associações entre os medicamentos por grupos farmacológicos

7

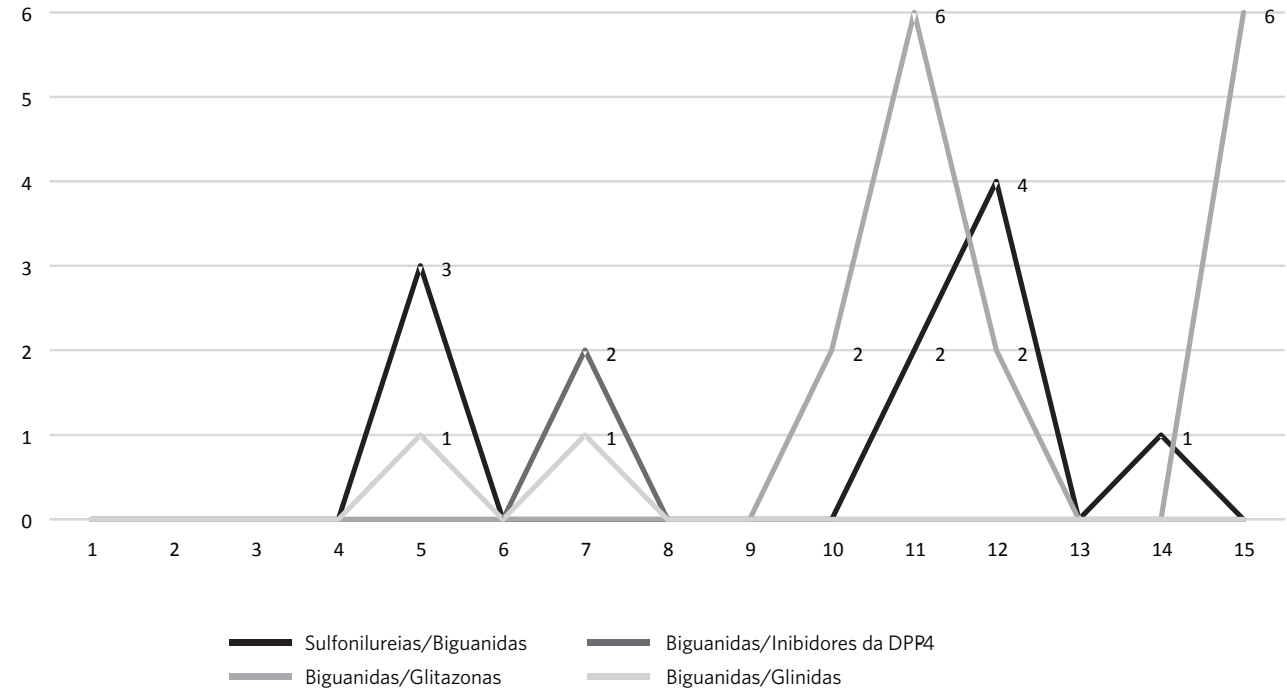

Fonte: Guimarães ${ }^{9}$ 
Verifica-se que o grupo Sulfonilureia, ao ser associado à Biguanida, permite a diminuição da $\mathrm{HbAlC}$ e da hipoglicemia grave, com baixo custo e ganho de peso para o paciente. Esses medicamentos são: glimepiride, associado à metformin hydrochloride; glyburide, associado à metformin hydrochloride ${ }^{10}$. Os princípios ativos metformin e metformin hydrochloride permitem outras associações.

a) Glitazonas - essa associação permite menor risco hipoglicêmico, diminuição da $\mathrm{HbAlC}$, com alto custo e ganho de peso para o paciente (formação de edemas). Fazem parte deste grupo a metformin hydrochloride e rosiglitazone maleate;

b) Inibidores da DPP-4 - a associação permite menor risco hipoglicêmico, diminuição intermediária da $\mathrm{HbAlC}$, com alto custo e sem influência no peso do paciente. Os medicamentos que fazem parte deste grupo são o linagliptin, saxagliptin hydrochloride monohydrate, sitagliptin phosphate monohydrate, vildagliptin e sitagliptin phosphate;

c) Glinidas - a associação permite a estimulação de secreção no sistema pancreático de insulina e sensibilização do sistema hepático para os hormônios hipoglicêmicos. Os medicamentos que fazem parte desta associação são a metformin e a nateglinide ${ }^{\mathbf{1 8}}$.

Destacam-se, também, as associações direcionadas à diminuição dos eventos hipoglicêmicos na fase intraprandial, em pacientes com baixa resposta à monoterapia insulínica; uma insulina de ação normal com uma de longa duração para controle dos níveis glicêmicos, entre a insulin aspart/insulin aspart protamine, insulin human recombinant/insulin isophane recombinant human ${ }^{10}$.

\section{Inovação nos medicamentos antidiabéticos}

Atualmente, as empresas utilizam a inovação, radical ou incremental, como uma estratégia para diferenciar e garantir a competitividade de seus produtos no mercado. $\mathrm{O}$ quadro 2, a seguir, apresenta a análise do tipo de inovação e ilustra os resultados do exame do tipo de inovação, apresentados nos medicamentos antidiabéticos, a partir dos princípios ativos, nos grupos farmacológicos, e a classificação do tipo de inovação.

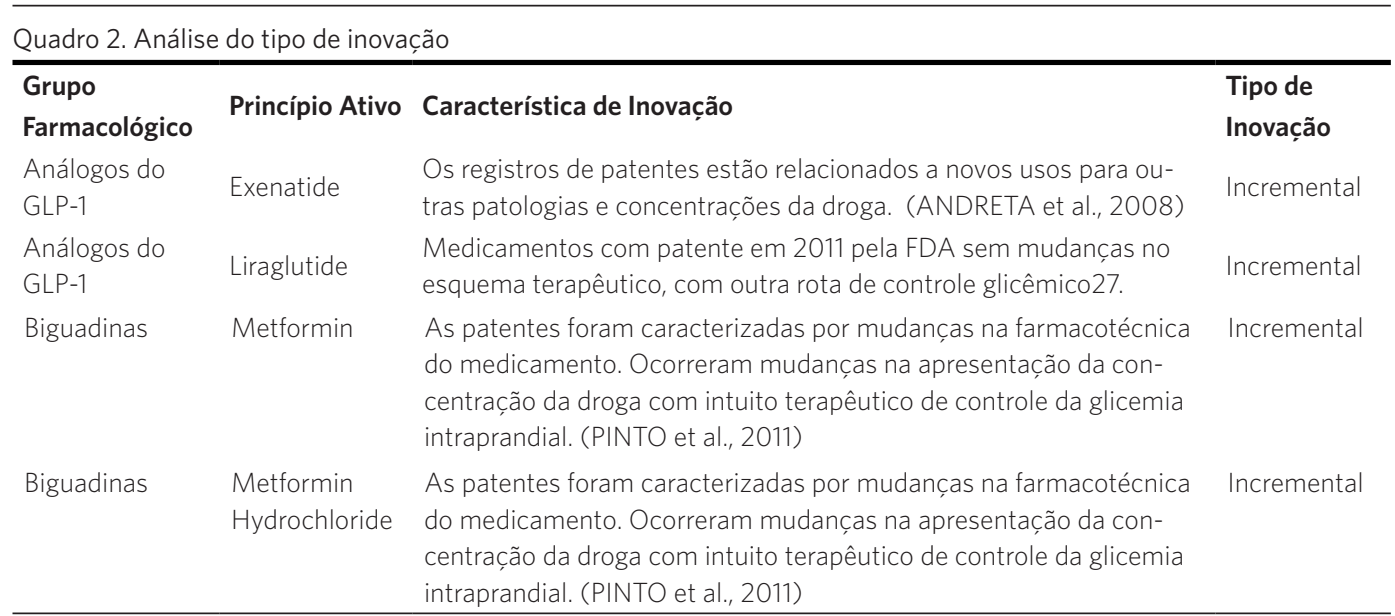




\begin{tabular}{|c|c|c|c|}
\hline \multicolumn{4}{|c|}{ Quadro 2. (cont.) } \\
\hline $\begin{array}{l}\text { Derivadas da } \\
\text { insulina }\end{array}$ & Insulin Aspart & $\begin{array}{l}\text { Análogo de ação rápida da insulina humana, modificação estrutural, } \\
\text { mesma farmacocinética e farmacodiâmica da insulin glulisine re- } \\
\text { combinant. As patentes são para uso de outras patologias além da } \\
\text { diabetes. (CONEGERO, 2011) }\end{array}$ & Incremental \\
\hline $\begin{array}{l}\text { Derivadas da } \\
\text { insulina }\end{array}$ & $\begin{array}{l}\text { Insulin } \\
\text { Glargine }\end{array}$ & $\begin{array}{l}\text { Uma administração diária de insulina tem a mesma eficácia anti- } \\
\text {-hiperglicêmica que múltiplas doses diárias de insulina NPH, com } \\
\text { uma menor frequência de episódios hipoglicêmicos em intervalos } \\
\text { maiores, período interprandial28. }\end{array}$ & Incremental \\
\hline $\begin{array}{l}\text { Derivadas da } \\
\text { insulina }\end{array}$ & $\begin{array}{l}\text { Recombinant } \\
\text { Glulisine } \\
\text { Insulin }\end{array}$ & $\begin{array}{l}\text { Análogo de ação rápida, modificação estrutural, mesma farmacoci- } \\
\text { nética e farmacodinâmica da insulin aspart. (KLAUS et al., 2006) }\end{array}$ & Incremental \\
\hline $\begin{array}{l}\text { Derivadas da } \\
\text { insulina }\end{array}$ & $\begin{array}{l}\text { Recombinant } \\
\text { Human Insulin }\end{array}$ & $\begin{array}{l}\text { BPH - insulina protamínica neutra de Hagedorn. (CONEGERO et al., } \\
\text { 2011) }\end{array}$ & Incremental \\
\hline $\begin{array}{l}\text { Derivadas da } \\
\text { insulina }\end{array}$ & $\begin{array}{l}\text { Biphasic } \\
\text { Insulin }\end{array}$ & $\begin{array}{l}\text { Análogo de ação rápida, modificação estrutural. (HOLMAN et al., } \\
\text { 2007) }\end{array}$ & Incremental \\
\hline $\begin{array}{l}\text { Derivadas da } \\
\text { insulina }\end{array}$ & $\begin{array}{l}\text { Insulin } \\
\text { Degludec }\end{array}$ & Insulina basal de ultraduração. (HOLMAN et al., 2007) & Incremental \\
\hline $\begin{array}{l}\text { Derivadas da } \\
\text { insulina }\end{array}$ & $\begin{array}{l}\text { Insulin } \\
\text { Detemir }\end{array}$ & $\begin{array}{l}\text { Uma administração diária de insulina tem a mesma eficácia anti- } \\
\text {-hiperglicêmica que múltiplas doses diárias de insulina NPH, com } \\
\text { uma menor frequência de episódios hipoglicêmicos em intervalos } \\
\text { intermediários, período interprandial. (HOLMAN et al., 2007) }\end{array}$ & Incremental \\
\hline $\begin{array}{l}\text { Derivadas da } \\
\text { insulina }\end{array}$ & $\begin{array}{l}\text { Recombinant } \\
\text { Lispro Insulin }\end{array}$ & $\begin{array}{l}\text { Primeiro análogo da insulina de ação rápida que está relacionado } \\
\text { com recentes associações medicamentosas com outros derivados e } \\
\text { grupos antidiabéticos. (PEREZ; RULL, 2005). }\end{array}$ & Incremental \\
\hline Glinidas & Nateglinide & $\begin{array}{l}\text { Os registros de patentes estão relacionados a novos usos para ou- } \\
\text { tras patologias, tais como: colite ulcerativa, síndrome pré-menstrual, } \\
\text { doenças cardiovasculares, doenças do tecido conjuntivo, desordem } \\
\text { metabólica, neoplasias, fibrose cística. (RAMALHO; LIMA, 2006). }\end{array}$ & Incremental \\
\hline Glinidas & Repaglinide & $\begin{array}{l}\text { Os registros de patentes estão relacionados a novos usos para ou- } \\
\text { tras patologias, tais como: distúrbios do metabolismo de lipídeos, } \\
\text { doença muscular, epilepsia, doença ocular, dislexia, disfasia, distonia, } \\
\text { fraqueza muscular, doença neurológica24. }\end{array}$ & Incremental \\
\hline Glitazonas & Rosiglitazone & $\begin{array}{l}\text { Registros de patente para novas patologias ou adequação de con- } \\
\text { centração da droga (desordem psiquiátrica, artrite, psoríase, asma, } \\
\text { colite ulcerativa, cisto do ovário). (SILVA; CASTRO, 2010). }\end{array}$ & Incremental \\
\hline Glitazonas & Pioglitazone & $\begin{array}{l}\text { Registro de patente para novas doenças, tais como: caqueixa, ano- } \\
\text { rexia nervosa, doença inflamatória intestinal, osteoporose, artrite, } \\
\text { aterosclerose, doença autoimune, síndrome da imunodeficiência } \\
\text { adquirida, doença de Alzheimer, artrite reumatoide, coma, desordem } \\
\text { consciência, doença cerebrovascular, doença respiratória, distúrbio } \\
\text { auditivo, hiperplasias. (BRANCHTEIN; MATOS, 2002). }\end{array}$ & Incremental \\
\hline Glitazonas & $\begin{array}{l}\text { Pioglitazone } \\
\text { Hydrochloride }\end{array}$ & $\begin{array}{l}\text { Registro de patente para novas doenças, tais como: caqueixa; ano- } \\
\text { rexia nervosa, doença inflamatória intestinal, osteoporose, artrite, } \\
\text { aterosclerose, doença autoimune, síndrome da imunodeficiência } \\
\text { adquirida, doença de Alzheimer, artrite reumatoide, coma, desordem } \\
\text { consciência, doença cerebrovascular, doença respiratória, distúrbio } \\
\text { auditivo, hiperplasias23. }\end{array}$ & Incremental \\
\hline $\begin{array}{l}\text { Inibidores da } \\
\text { DPP-4 }\end{array}$ & Linagliptin & $\begin{array}{l}\text { Registro de patente associado à obesidade, arterosclerose, anorexia, } \\
\text { Sida, doenças autoimunes, infertilidade, ovário cisto, desordem do } \\
\text { crescimento, doença inflamatória intestinal, obesidade, anorexia } \\
\text { nervosa, osteoporose, artrite, aterosclerose, doença autoimune, } \\
\text { síndrome da imunodeficiência adquirida, hiperinsulina, não insulino- } \\
\text { dependente, diabetes mellitus, complicação diabética. (TASKINEN } \\
\text { et al., 2011) }\end{array}$ & Incremental \\
\hline
\end{tabular}




\begin{tabular}{|c|c|c|c|}
\hline \multicolumn{4}{|c|}{ Quadro 2. (cont.) } \\
\hline $\begin{array}{l}\text { Inibidores da } \\
\text { DPP-4 }\end{array}$ & Saxagliptin & $\begin{array}{l}\text { Registro de patente associado à obesidade, arterosclerose, anorexia, } \\
\text { Sida, doenças autoimunes, infertilidade, ovário cisto, desordem do } \\
\text { crescimento, doença inflamatória intestinal, obesidade, anorexia } \\
\text { nervosa, osteoporose, artrite, aterosclerose, doença autoimune, } \\
\text { síndrome da imunodeficiência adquirida, hiperinsulinemia, não } \\
\text { insulinodependente, diabetes mellitus, complicações diabéticas. } \\
\text { (ROSENSTOCK et al., 2009) }\end{array}$ & Incremental \\
\hline $\begin{array}{l}\text { Inibidores da } \\
\text { DPP-4 }\end{array}$ & $\begin{array}{l}\text { Sitagliptin } \\
\text { Phosphate } \\
\text { Monohydrate }\end{array}$ & $\begin{array}{l}\text { Registro de patente associado à obesidade, arterosclerose, anorexia, } \\
\text { Sida, doenças autoimunes, infertilidade, ovário cisto, desordem do } \\
\text { crescimento, doença inflamatória intestinal, obesidade, anorexia } \\
\text { nervosa, osteoporose, artrite, aterosclerose, doença autoimune, } \\
\text { síndrome da imunodeficiência adquirida, hiperinsulinemia, não insu- } \\
\text { linodependente, diabetes mellitus, complicações diabéticas. (SILVA; } \\
\text { CASTRO, 2010). }\end{array}$ & Incremental \\
\hline $\begin{array}{l}\text { Inibidores da } \\
\text { DPP-4 }\end{array}$ & Vildagliptin & $\begin{array}{l}\text { Registro de patente associado à obesidade, arterosclerose, anorexia, } \\
\text { Sida, doenças autoimunes, infertilidade, ovário cisto, desordem do } \\
\text { crescimento, doença inflamatória intestinal, obesidade, anorexia } \\
\text { nervosa, osteoporose, artrite, aterosclerose, doença autoimune, } \\
\text { síndrome da imunodeficiência adquirida, hiperinsulinemia, não } \\
\text { insulinodependente, diabetes mellitus, complicações diabéticas. } \\
\text { (ALEXANDER et al., 2008). }\end{array}$ & Incremental \\
\hline $\begin{array}{l}\text { Inibidores da } \\
\alpha \text {-glicose }\end{array}$ & Acarbose & $\begin{array}{l}\text { Foi identificado como incremental na sua formulação ou apresen- } \\
\text { tação em novas concentrações e caracterização do processo de } \\
\text { obtenção da acarbose como suplemento no tratamento da diabetes, } \\
\text { bem como uso para outras patologias gastrointestinais. (INZUCCHI, } \\
\text { 2002). }\end{array}$ & Incremental \\
\hline Sufanilureias & Gliclazide & $\begin{array}{l}\text { As patentes foram caracterizadas por mudanças na farmacotécnica } \\
\text { do medicamento (formas de liberação retardada e prolongada). } \\
\text { Ocorreram mudanças na apresentação da concentração da dro- } \\
\text { ga com intuito terapêutico de controle da glicemia intraprandial. } \\
\text { (BRANCHTEIN; MATOS, 2006). }\end{array}$ & Incremental \\
\hline Sufanilureias & Glimepiride & $\begin{array}{l}\text { As patentes foram apresentadas para doenças associadas a diabe- } \\
\text { tes, hipertensão, obesidade, resistência à insulina, desequilíbrio do } \\
\text { metabolismo lipídico. (ARAUJO; BRITO; CRUZ, 2000). }\end{array}$ & Incremental \\
\hline Sufanilureias & Glipizide & $\begin{array}{l}\text { As patentes foram apresentadas para doenças associadas a diabe- } \\
\text { tes, hipertensão, obesidade, resistência à insulina, desequilíbrio do } \\
\text { metabolismo lipídico. (GONZALEZ; PEREZ, 2008). }\end{array}$ & Incremental \\
\hline Sufanilureias & Glyburide & $\begin{array}{l}\text { As patentes foram apresentadas para doenças associadas a diabe- } \\
\text { tes, hipertensão, obesidade, resistência à insulina, desequilíbrio do } \\
\text { metabolismo lipídico. (GONZALEZ; PEREZ, 2008). }\end{array}$ & Incremental \\
\hline
\end{tabular}

Fonte: Elaboração própria, com base em Guimarães?

Na análise do tipo de inovação ocorrida nos princípios ativos, no período de 1998 a 2012, constatou-se que não houve inovação radical. As mudanças ocorridas no grupo dos Análogos do GLP-1 referem-se aos novos usos do medicamento em outras patologias, concentração da droga e medicamento com patente em 2011, pela Food and Drug Administration (FDA), sem mudanças no esquema terapêutico com outra rota de controle glicêmico.
No grupo da Biguanidas, a inovação refere-se às mudanças ocorridas na apresentação da concentração da droga e na apresentação do medicamento na forma de cápsula, comprimido e pó.

Quanto aos 'derivados da insulina', a inovação é proveniente do uso em outras patologias; menor frequência de episódios hipoglicêmicos em intervalos maiores no período interprandial, síntese de análogos de 
ação rápida e de ultralonga duração através de uma administração diária na busca de diminuir a frequência de episódios hipoglicêmicos em intervalos intermediários.

No grupo dos Glinidas, Glitazonas e Inibidores da DPP-4, os registros de patentes estão relacionados a novos usos para outras patologias.

Para os inibidores da $\alpha$-glicosidase, ocorreu a apresentação em novas concentrações e caracterização do processo de obtenção da acarbose como suplemento no tratamento da diabetes, bem como em outras patologias.

Por fim, no grupo das Sulfonilureias, as patentes foram caracterizadas por mudanças nas formas de liberação retardada e prolongada, mudanças na apresentação da concentração da droga e para doenças associadas à diabetes, tais como hipertensão, obesidade, resistência à insulina, desequilíbrio do metabolismo lipídico.

\section{Trajetórias tecnológicas dos medica- mentos genéricos, antidiabéticos}

A partir da análise do processo de inovação, foram traçadas as trajetórias tecnológicas dos medicamentos genéricos, antidiabéticos, apresentadas no gráfico 3 .

Gráfico 3. Medicamentos por grupos farmacológicos e lançamentos no Brasil

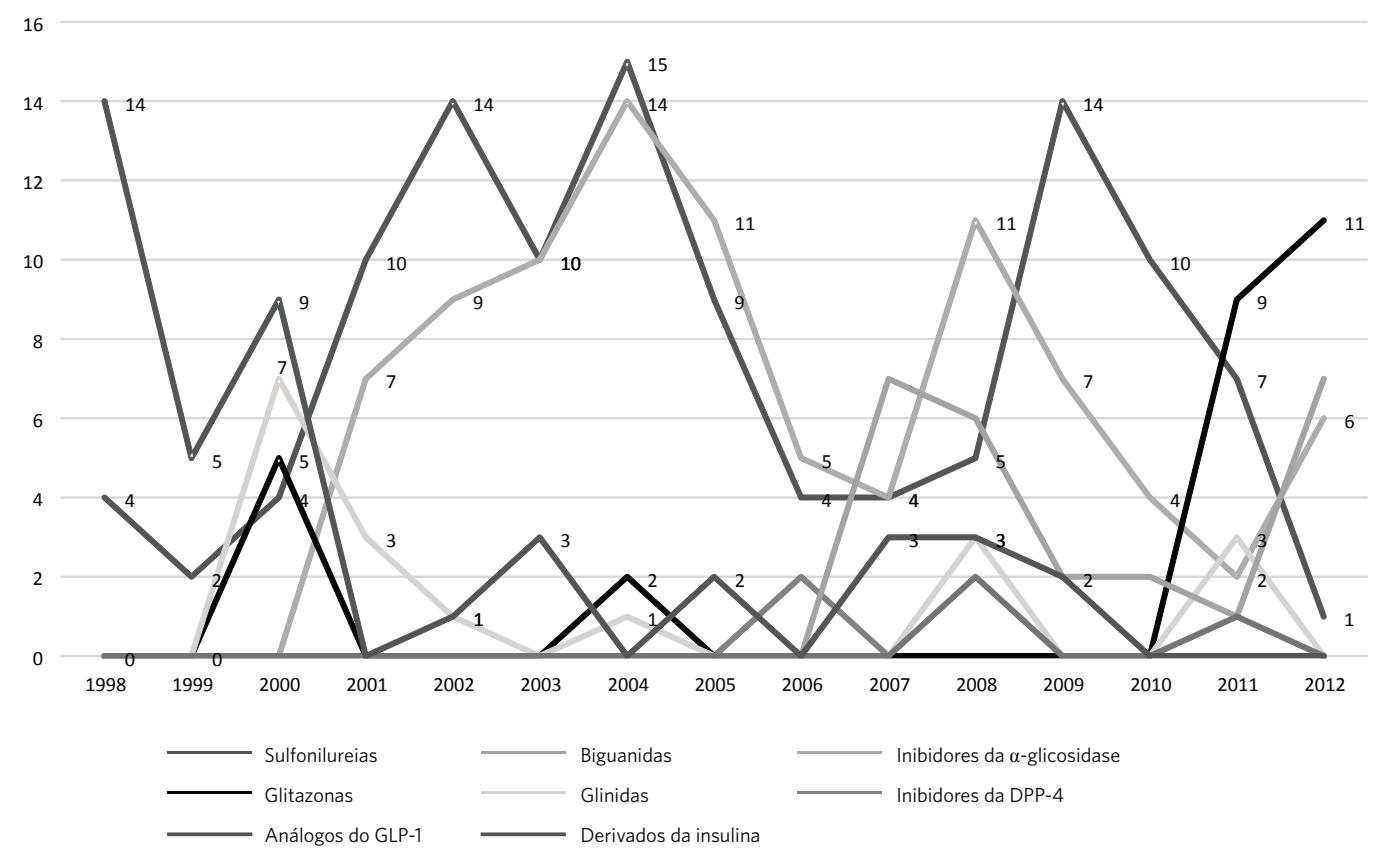

Fonte: Guimarães ${ }^{9}$

O gráfico 3 apresenta os medicamentos e seus respectivos números de lançamentos no Brasil, no período de 1998 a 2012, na trajetória correspondente. No entanto, as duas trajetórias começaram a apresentar sinais de esgotamento nos anos 2000. Entre os anos de 2000 e 2005 , foram aprovados 104 biofármacos no mercado; e de 2006 a 2009, quando se ampliou a desaceleração da introdução de novas tecnologias, foram aprovados 25 . 
A despeito da desaceleração, esse segmento tem ampliado sua participação na indústria em função do seu elevado custo. Nos anos de 2000 e 2009, a participação desses produtos nas vendas da indústria farmacêutica atingiu
$13 \%$, em 2009, contra $6,5 \%$, em $2000^{11,12}$.

No quadro 3, abaixo, podem-se verificar os medicamentos genéricos, lançados no Brasil, de acordo com a sua trajetória tecnológica.

Quadro 3. Medicamentos lançados ao longo das trajetórias tecnológicas

\begin{tabular}{l}
\hline Trajetória tecnológica baseada em química fina \\
\hline Acarbose, chlorpropamide, exenatide, gliclazide, glimepiride, glipizide, glucagon, glyburide, linagliptin, liraglutide, \\
metformin, metformin hydrochloride, nateglinide, pioglitazone hydrochloride, repaglinide, rosiglitazone maleate, \\
saxagliptin hydrochloride, sitagliptin phosphate monohydrate, vildagliptin, metformin hydrochloride/rosiglitazone \\
maleate, metformin hydrochloride/saxagliptin hydrochloride, metformin hydrochloride/sitagliptin phosphate \\
monohydrate, metformin hydrochloride/vildagliptin, glimepiride/metformin hydrochloride, glyburide/metformin \\
hydrochloride. \\
\hline Trajetória tecnológica baseada em biotecnologia moderna \\
\hline Insulin aspart, insulin detemir, insulin glargine, insulin glulisine recombinant, insulin human recombinant, insulin \\
isophane recombinant human, insulin lispro recombinant, insulin aspart/insulin aspart protamine, insulin human \\
recombinant/insulin isophane recombinant human. \\
\hline Fonte: Elaboração própria.
\end{tabular}

\section{Discussão}

Em função da imaturidade do sistema de inovação brasileiro, as empresas farmacêuticas nacionais não são líderes no processo de inovação. No caso dos antidiabéticos, as patentes aqui depositadas são de grandes corporações internacionais. Apesar dos recentes esforços em articular a Política de Saúde com as Políticas Industriais e Tecnológicas e de Ciência e Tecnologia (C\&T), de modo a garantir o equilíbrio entre a lógica sanitária, voltada para o atendimento de necessidades, prestação de uma atenção com qualidade, efetividade e equidade ${ }^{13}$; e a lógica econômica, baseada no lucro, os reflexos em termos de novos produtos ainda são escassos.

Um conhecimento novo pode propiciar uma inovação que apresente uma utilidade econômica para a sociedade. No Brasil, historicamente, ocorreu uma desconexão entre a política científica e tecnológica e as políticas de saúde. Isso se traduz em um descompasso entre a produção de conhecimento e sua aplicação ${ }^{7,14}$.
Esse comportamento contribui para que a capacidade de inovação do segmento seja incipiente e reforça a dependência externa de bens voltados para a saúde.

Para o controle da diabetes, novas tecnologias, produtos e serviços são lançados diariamente no mercado, e novos processos são incorporados nos serviços de saúde, guiados pela lógica do grande capital internacional, que fortalece o modelo médico hegemônico de atenção adotado, curativo e intensivo em tecnologias materiais. As tecnologias geradas são, em geral, agregativas, e não substitutivas, sendo que os critérios de obsolescência são complexos, e as demandas pressionam os sistemas de saúde.

Portanto, a Organização do Sistema de Saúde é um elemento estratégico no setor saúde, ao apontar que direção a inovação deve seguir. Neste sentido, a despeito de uma série de políticas voltadas para a promoção e a prevenção, observa-se uma tensão crescente entre as lógicas sanitária e econômica, decorrentes da introdução 
das sucessivas inovações no mercado. $\mathrm{Ou}$ seja, quando diferentes necessidades concorrem pelos mesmos recursos, as necessidades deveriam ser subordinadas à política nacional de saúde, em suas diferentes esferas. No entanto, dada a estrutura de mercado fortemente oligopolizada da indústria farmacêutica, essas tecnologias têm sido incorporadas rapidamente pelo sistema de saúde.

Portanto, a PNM é um instrumento fundamental para a promoção da assistência à saúde da população e para garantir segurança, eficácia e qualidade dos medicamentos. Entretanto, é preciso garantir uma política de medicamentos de interesse para a saúde. Isto é, que assegure a integralidade de forma efetiva e segura, porém, sem comprometer a sustentabilidade do sistema ${ }^{\mathbf{1 2}}$.

É preciso buscar mecanismos de controle dos laboratórios para assegurar que tanto o poder público quanto os cidadãos não fiquem sujeitos à vontade privada. Talvez, a intensificação de parcerias com o setor privado possa ser favorável à inovação no País.

\section{Mudança de paradigma no cuidado da diabetes}

Para o cuidado da diabetes, observou-se que as inovações continuam baseadas na utilização de propostas medicamentosas que utilizam os mesmos conceitos clínicos de controle do índice glicêmico intracelular e vascular dos últimos 14 anos $^{\mathbf{1 4}}$.

Não houve inovação radical no período. As inovações radicais e incrementais estão associadas ao surgimento de um paradigma tecnológico que se iniciou com a ascensão e a consolidação da química fina, no final do século XIX, e da biotecnologia, no final do século XX. Trata-se de duas trajetórias distintas que levam à criação de um padrão de produção muito semelhante em sua finalidade. Portanto, não houve a consolidação de um novo paradigma. Não houve uma redução de custos ou um crescimento rápido na oferta, com potencial para uso ou incorporação dessa tecnologia em vários processos e produtos no sistema econômico, mudando a configuração da estrutura produtiva hegemônica ${ }^{14}$.

A inovação ocorrida no tratamento da diabetes caracteriza-se pela perspectiva de inclusão de novos protocolos clínicos e adequações posológicas dos grupos de fármacos reconhecidos como antidiabéticos, medicamentos de controle glicêmico e insulinas recombinantes ${ }^{\mathbf{1 4}}$, e, também, na busca de novos alvos metabólicos efetivos para o tratamento da diabetes.

Tais tendências na pesquisa sobre o cuidado da diabetes estão exemplificadas no estudo de enzimas ativadoras do desempenho glicêmico ou receptores de ácidos graxos livres ${ }^{15-17}$.

Outros caminhos envolvem genes como Wnt, NAD +, precursores da nicotinamida, fatores de transcrição da classe ' $\mathrm{O}$ ', citocinas e fatores de crescimento de Eritropoietina (EPO). Cada um deles determina o desenvolvimento, a sobrevivência e os mecanismos de lesão e longevidade celular na diabetes ${ }^{18}$.

Entre as propostas do cuidado com a diabetes, todas estão relacionadas ao perfil e à resposta glicêmica tecidual do paciente, estabelecendo adequações medicamentosas com associações entre os medicamentos antidiabéticos e insulinas recombinantes disponíveis no mercado. Essas convergências nas inovações metabólicas, acima citadas, estão relacionadas à modulação da homeostase da glicose e à sensibilidade da insulina, demonstrando uma estratégica clínica de aprimoramento aos tratamentos abordados no controle glicêmico, com os medicamentos e a terapia insulínica existentes e compreensão de novas rotas de adequação glicêmica da diabetes, sem mudanças de paradigmas tecnológicos no seu tratamento, uma vez que não houve o surgimento de um problema científico que não pudesse ser resolvido pelos instrumentos já existentes. 


\section{Trajetórias tecnológicas: possíveis direções a seguir}

No âmbito da indústria farmacêutica, duas trajetórias distintas convivem e disputam o mercado.

Uma baseada na química fina, com origem no final do século IX e que engendrou os fármacos tradicionais, e aquela nascida da biologia molecular que começa a se desenvolver a par-

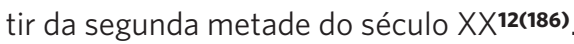

No caso dos antidiabéticos, a introdução de inovações radicais talvez seja ainda mais tímida. $\mathrm{Na}$ análise dos princípios ativos dos 26 antidiabéticos lançados no período, verificou-se que todos apresentaram inovações incrementais, ou seja, incorporaram pequenas alterações nas características técnicas dos medicamentos, não se configurando o desenvolvimento de um novo produto.

Confirma-se, desse modo, que nesse tipo de inovação as diferenças apresentadas podem estar nas características técnicas, na qualidade, na produtividade, na eficiência técnica, nos custos, pois resultam de um conjunto de melhorias sucessivas a que estão submetidas todas as tecnologias com o uso, não implicando o surgimento de novos produtos ou processos ou a exploração de novos mercados $^{\mathbf{1 2 , 1 9}}$.

Apesar de o Brasil ser um país com grandes desigualdades sociais, é importante garantir aos usuários do Sistema Único de Saúde (SUS) o acesso às inovações tecnológicas em saúde disponíveis no mercado e, assim, garantir o cuidado integral. Para Merhy ${ }^{20}$, o cuidado integral ocorreria a partir de uma combinação generosa e flexível de tecnologias duras, leve-duras e leves. Nesse sentido, o Ministério da Saúde exerce grande influência nos percursos da incorporação. Apesar da sua fraca atuação no percurso científico, ao deter o poder de compra da tecnologia, é responsável pela concessão do registro dos medicamentos, por diretrizes terapêuticas, negociação de preços, aquisição e distribuição de medicamentos. Além disso, é de responsabilidade do MS regulamentar o processo de incorporação de tecnologias ao SUS, de modo a atender às demandas de saúde, o que pressupõe levar em conta tanto as questões de risco e efetividade quanto às políticas e de custo.

\section{Conclusões}

Tem sido um grande desafio para os sistemas de saúde no mundo cuidar da população com alta prevalência de diabetes, frente a uma crescente demanda por procedimentos diagnósticos e terapêuticos desses pacientes, principalmente na saúde, onde bens são gerados num conjunto de segmentos produtivos que compartilham um elevado grau de inovação, em que a principal estratégia para o retorno do investimento e para a lucratividade é a proteção patentária. Fato que implica elevados preços de entrada dessas tecnologias no mercado, inclusive após a expiração das patentes, tendo em vista os elevados investimentos em marketing.

A base produtiva desses bens e serviços é relevante na geração, incorporação e difusão de inovação, dado que as indústrias são intensivas em ciência e tecnologia. No Brasil, a capacidade de inovação do setor de saúde é limitada às áreas mais distantes da fronteira tecnológica, sendo marcante a produção de medicamentos genéricos, ou seja, aqueles cujas patentes expiraram. Isso decorre da falta de conexão entre as políticas científica e tecnológica e a política de saúde, apesar do Complexo Econômico Industrial da Saúde (Ceis) ser reconhecido como um segmento importante para o desenvolvimento econômico. Portanto, o descompasso entre a produção de conhecimento e sua aplicação reflete dependência externa de bens voltados para a saúde, sendo o perfil industrial no Brasil composto pelo segmento de produção nacional público, nacional privado e de empresas de capital transnacional. 
Articular as políticas de saúde e de C\&T às políticas industrial e comercial reduz a dependência tecnológica na área de saúde e possibilita a pesquisa e o desenvolvimento de bens e serviços, visando a atender às necessidades de saúde da população brasileira.

No âmbito do SUS, é imprescindível definir o padrão tecnológico. Ou seja, definir as tecnologias mais seguras, mais eficazes e que podem favorecer a implantação de um modelo integral de atenção à saúde. No que tange ao cuidado da diabetes, a oferta de tecnologias tradicionais, associadas às estratégias preventivas, tem se traduzido em melhorias nos indicadores de morbidade sem grandes impactos para o orçamento da saúde. Por seu turno, a criação de capacidade científica interna, especialmente pelos laboratórios públicos, pode constituir uma estratégia importante para as tecnologias de base biotecnológica, que têm respondido por parcelas significativas dos gastos com medicamentos do SUS (em torno de $5 \%$, em termos de quantidade, e quase $50 \%$ em valor monetário) ${ }^{\mathbf{1 2}}$.

Para isso, é fundamental levar este debate para o campo da saúde coletiva. Para o projeto da Reforma Sanitária, no Sistema
Universal de Saúde, é imprescindível garantir uma proposta de desenvolvimento tecnológico coerente com os princípios do SUS.

\section{Colaboradores}

Guimarães JMM contribuiu para a concepção e o planejamento, análise e interpretação dos dados; elaboração do rascunho, revisão crítica do conteúdo e aprovação da versão final do manuscrito. Aragão ES contribuiu para a interpretação dos dados, elaboração do rascunho, revisão crítica do conteúdo e aprovação da versão final do manuscrito. Galdino FSS contribuiu com as seguintes atividades: interpretação dos dados, elaboração do rascunho e revisão crítica do conteúdo, e aprovação da versão final do manuscrito. Almeida BA contribuiu para a interpretação dos dados, elaboração do rascunho, revisão crítica do conteúdo e participou da aprovação da versão final do manuscrito. Silva SALS contribuiu para a interpretação dos dados, elaboração do rascunho, revisão crítica do conteúdo e aprovação da versão final do manuscrito.

\section{Referências}

1. Brasil. Portaria $\mathrm{n}^{\mathrm{o}} 2.583$, de 10 de outubro de 2007 . Define elenco de medicamentos e insumos disponibilizados pelo Sistema Único de Saúde, nos termos da Lei $n^{\circ} 11.347 / 2006$, aos usuários portadores de Diabetes Mellitus. Diário Oficial da União. 10 Out 2007
2. Dosi G. Mudança Técnica e Transformação Industrial: a teoria e uma aplicação à Indústria dos Semicondutores. São Paulo: Unicamp; 2006.

3. Brasil. Ministério da Saúde. Diabetes Mellitus. Brasília, DF: MS; 2006. 
4. Schumpeter J. A. Teoria do desenvolvimento econômico: uma investigação sobre lucros, capital, crédito, juro e o ciclo econômico. São Paulo: Nova Cultural; 1997.

5. Orsenigo L, Dosi G, Mazzucato M. The dynamics of knowledge accumulation, regulation and appropriability in the pharma-biotech sector: some policy issues. In: Mazzucato M, Dosi G, editores. Knowledge accumulation and industry evolution: pharma-biotech. Cambridge University Press: UK; 2006. p. $402-431$.

6. Malerba F, Orsenigo L. Innovation and market structure in the dynamics of the pharmaceutical industry and biotechnology: towards a history-friendly model, Industrial and Corporate Change. In: Winter Conference; 2001 jun 12-15. Aalborg: Druid; 2001.

7. Aragão E, Loureiro S, Temporão JG. Trajetórias tecnológicas na indústria farmacêutica: desafios para a equidade no Brasil. In: Paim JS, Almeida Filho N organizadores. Saúde Coletiva: Teoria e Prática. Rio de Janeiro: Medbook; 2014.

8. Kunh T. A Estrutura das Revoluções Científicas. 5. ed. São Paulo: Perspectiva; 2009.

9. Guimarães JMM. Paradigmas e trajetórias tecnológicas em saúde: mídia, acesso e cuidado do diabetes, [tese]. Salvador: Universidade Federal da Bahia, Instituto de Saúde Coletiva; 2014.

10. Sociedade Brasileira de Diabetes. Diretrizes da Sociedade Brasileira de Diabetes. 3. ed. Itapevi: SBD; 2009.

11. Walsh G. Biopharmacetiucal Approval Trends: 2009. BioPharm International. 2010; 23(10).

12. Aragão E. Redes de inovação: o caso do segmento de biotecnologias para diagnóstico voltadas a saúde humana [tese]. Salvador: Universidade Federal da Bahia, Instituto de Saúde Coletiva; 2011.
13. Paim JS. Modelos assistenciais: reformulando o pensamento e incorporando a proteção e a promoção da saúde. In: Anais dos Seminários Temáticos Permanentes. 2001 mar 23. Brasília, DF: UFBA/ISC; 2001

14. Lopes VP, Santos Júnior MC, Santos Júnior AF, et al. Farmacologia do diabetes mellitus tipo 2: antidiabéticos orais, insulina e inovações terapêuticas. Rev Eletrôn Farmácia. 2012; 9(3):69-90.

15. Freeman C, Perez C. Structural crises of adjustmet: business cycles and investment behaviour. In: Dosi G. Techincal change and economic theory. Londres: Printer Publishers; 1988.

16. Bordone L, Guarente L. Calorie restriction, SIRT1 and metabolism: understanding longevity. Nat Rev Mol Cell Biol. 2005 abr; 6(4):298-305.

17. Lazar DF, Saltiel AR. Lipid phosphatases as drug discovery targets for type 2 diabetes. Nat Rev Drug Discov. 2006 abr [acesso em 2013 out 10]; 5(4):333342. Disponível em: http://www.nature.com/nrd/ journal/v5/n4/abs/nrd2007.html

18. Zhang S, Zhang ZY. PTP1B as a drug target: recent developments. In PTP1B inhibitor discover. Biochemistry Mol Biol. 2007 maio; 12(9-10):373-381.

19. Caetano R. Paradigmas e trajetórias do processo de inovação tecnológica em saúde. Physis. 1998; 8(2):71-94.

20. Merhy EE. Em busca do tempo perdido: a micropolítica do trabalho vivo em saúde. In: Merhy EE, Onocko R, organizadores. Agir em saúde: um desafio para o público. 3. ed. São Paulo: Hucitec; 2007.

Recebido em 14/07/2018 Aprovado em 18/09/2018

Conflito de interesses: inexistente

Suporte financeiro: não houve 